\title{
Knotted threads of darkness
}

\section{Dark lines within a laser beam can be manipulated to form stable vortex knots.}

D estructive interference may lead to complete cancellation when light waves travelling in different directions cross, and in three-dimensional space this occurs along lines that are vortices of electromagnetic energy flow ${ }^{1}$. Here we confirm theoretical predictions ${ }^{2,3}$ by experimentally creating combinations of optical laser beams in which these dark threads form stable loops that are linked and knotted.

Since Kelvin proposed his vortex atom hypothesis ${ }^{4}$, temporally persistent vortex knots have been sought in fluid mechanics ${ }^{5,6}$, superfluid flow ${ }^{7}$, field theory ${ }^{8}$ and nonlinear excitable media ${ }^{9}$. The optical vortices that we synthesize here are embedded in light beams created by a single-frequency laser (Fig. 1a; for methods, see supplementary information). A direct consequence of the linearity of Maxwell's equations for the electromagnetic field is that the intensity pattern in free space is static, so our optical vortices are stable in both space and time.

The optical phase cannot be defined on these dark threads: it is singular there (like the angle of longitude at the North Pole, for example). Consequently, there is a circulation of electromagnetic energy around the optical vortex. Around such a singularity, the phase changes by an integer multiple of $2 \pi$; this integer, $m$, is the strength of the singularity.

We combine Laguerre-gaussian beams ${ }^{10}$, which have an optical vortex of integer strength $m$ along the beam axis and two further variable parameters: $p$, the discrete radial number (the number of nodal rings about the beam axis), and $w$, the continuous waist width (which sets the transverse scale of the beam). These additional degrees of freedom allow us to create the same basic structures as those proposed in refs 2 and 3, but with the vortices more clearly defined by the surrounding intensity.

The loops, links and knot structures arise from the coaxial superposition of four beams. Three of these beams have an on-axis vortex with the same strength $m$, but different values of $p$ and $w$. The amplitudes are chosen so that both the field and its radial derivative are zero at a specific radius in the focal plane. The resulting destructive interference forms a dark loop around the axial strength singularity. Adding a simple gaussian beam with small amplitude distorts this loop into an $(m, 2)$ torus knot $^{11}$, threaded by an $m$-stranded helix of strength-one vortices. If $m=2$, the vortices form two linked loops (Fig. 1b); if $m=3$, they form a trefoil knot (Fig. 1c).

Our optical beams are produced by using a single-frequency helium-neon laser beam illuminating a hologram. This forms the

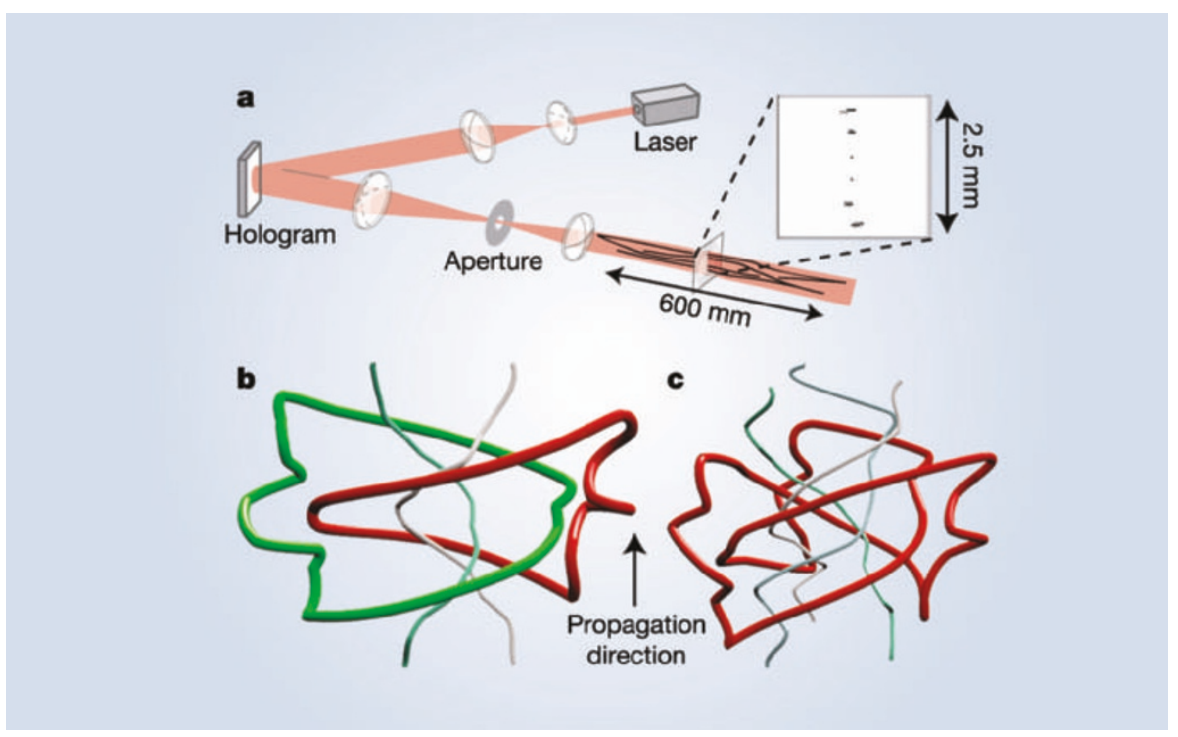

Figure 1 Knotted lines of darkness. a, Light from a hologram is passed through a spatial filter and a camera images the beam cross section in various planes. Thin black lines represent the threads of darkness that form the link or knot. Inset shows the observed oversaturated beam cross-sections at the beam waist of the link; dark points indicate positions where the optical vortex threads cross the observation plane. b,c, Three-dimensional representations of measured link (b) and trefoil knot (c) configurations. The link and knot are threaded by further vortices (represented by the thinner tubes) that follow the axis of the light beam.

intensity and phase structure of the desired superposition of Laguerre-gaussian beams. As the fine structure of the singularities lies in regions of near darkness, it is necessary to oversaturate the camera to determine their positions in the beam cross-section accurately. The resulting image contains points of darkness corresponding to the positions of the dark threads as they intersect the plane of the camera.

We have produced stable link and knot structures formed from vortices in an optical beam. Our observations demonstrate the precision control of light beams that is required to create complex regions of zero light intensity. Such regions could be used, for example, to confine cold atoms and BoseEinstein condensates in complex topologies ${ }^{12}$. Jonathan Leach ${ }^{\star}$, Mark R. Dennis $\dagger \neq$, Johannes Courtial ${ }^{\star}$, Miles J. Padgett ${ }^{\star}$ ${ }^{*}$ Department of Physics and Astronomy,

University of Glasgow, Glasgow G12 8QQ, UK e-mail: j.leach@physics.gla.ac.uk

$\dagger$ H. H. Wills Physics Laboratory, Tyndall Avenue,

Bristol BS8 1TL, UK

$\$$ School of Mathematics, University of

Southampton, Highfield SO17 1BJ, UK

1. Nye, J. F. \& Berry, M. V. Proc. R. Soc. Lond. A 336, 165-190 (1974).

2. Berry, M. V. \& Dennis, M. R. Proc. R. Soc. Lond. A 457, 2251-2263 (2001).

. Berry, M. V. \& Dennis, M. R. J. Phys. A 34, 8877-8888 (2001).

4. Thompson, W. Phil. Mag. 34, 15-24 (1867).

5. Moffatt, H. K. J. Fluid Mech. 35, 117-129 (1969).

6. Ricca, R. L., Samuels, D. C. \& Barenghi, C. F. J. Fluid Mech. 391, 29-44 (1999).

7. Poole, D. R., Scoffield, H., Barenghi, C. F. \& Samuels D. C. J. Low Temp. Phys. 132, 97-117 (2003).
8. Fadeev, L. \& Niemi, A. J. Nature 387, 58-61 (1997)

9. Winfree, A. T. Nature 371, 233-236 (1994).

10. Allen, L., Beijersbergen, M. W., Spreeuw, R. J. C. \&

Woerdman, J. P. Phys. Rev. A 45, 8185-8189 (1992).

11. Adams, C. C. The Knot Book (Freeman, New York, 1994).

12. Ruostekoski, J. \& Anglin, J. R. Phys. Rev. Lett. 86, 3934-3936 (2001).

Supplementary information accompanies this communication on Nature's website.

Competing financial interests: declared none.

\section{Plant genetics}

\section{Gene transfer from parasitic to host plants}

lant mitochondrial genes are transmitted horizontally across mating barriers with surprising frequency, but the mechanism of transfer is unclear ${ }^{1,2}$. Here we describe two new cases of horizontal gene transfer, from parasitic flowering plants to their host flowering plants, and present phylogenetic and biogeographic evidence that this occurred as a result of direct physical contact between the two. Our findings complement the discovery that genes can be transferred in the opposite direction, from host to parasite plant ${ }^{3}$.

Both cases of horizontal gene transfer involve the mitochondrial gene atp 1 and the recipient Plantago, a large cosmopolitan genus comprising mostly weeds. We examined 43 Plantago species, all containing an intact atpl gene. Despite the exceptional divergence of these genes, their relationships 
to one another (Fig. 1, top) and their inclusion in Plantaginaceae agree with independent estimates ${ }^{4}$ of the phylogeny for these taxa. This clade of Plantago atp1 genes is therefore most likely to be the product of standard vertical transmission.

Each of three related Plantago species contains a second, full-length atp 1 copy with several frameshift mutations. This clade of pseudogenes is allied with the distantly related genus Cuscuta, as indicated by bootstrap $(97 \%)$ and other analyses (Fig. 1, and see supplementary information), implying that a species of Cuscuta transferred a copy of atp 1 to the common ancestor of these three Plantago species. Cuscuta is a large genus of flowering plants (also known as dodders; Fig. 2) that parasitizes an unusually broad range of hosts ${ }^{5}$, including Plantago ${ }^{6}$. The three Plantago species are native to Europe and north Africa, as are many dodders, including C. europaea (Fig. 1).

Two other species of Plantago ( $P$. rigida and $P$. tubulosa) also contain a second, fulllength copy of atp1. These too are pseudogenes and are probably also derived by horizontal gene transfer. Bartsia, a genus in the parasitic family Orobanchaceae, is identified as the likely gene donor by phylogenetic analysis (81\% bootstrap support; Fig. 1). Biogeography neatly supports this inference, because $P$. rigida, $P$. tubulosa and most species of Bartsia are found exclusively at high altitudes in the northern Andes ${ }^{7,8}$.

Both lineages of Plantago atp1 pseudogenes were almost certainly acquired by horizontal gene transfer, as indicated by evidence against their inclusion in the Plantagowide clade of vertically transmitted atp 1 genes and in favour of their phylogenetically anomalous inclusion with parasitic plants. (The possibility of DNA contamination can be ruled out; see supplementary information.) Phylogenetic distribution of the transferred genes and molecular clock analysis (see supplementary information) indicate that both transfers were recent (within the past few million years).

Both transfers probably occurred by direct plant-to-plant transmission of DNA from parasitic to host plants. This is indicated by phylogenetic evidence that parasitic plants, which penetrate host plants intracellularly as part of their normal life cycle, served as donors in both transfers. It is also suggested by the striking biogeographic concordance of donors and recipients in the case of the high-altitude, northern Andean Bartsia, $P$. rigida and $P$. tubulosa, as well as by the broad host ranges of both groups of parasitic donors. The well documented exchange in both directions of macromolecules, viruses and phytoplasmas between parasitic plants and their hosts ${ }^{9-11}$ is also consistent with plant-to-plant transmission.

We cannot altogether rule out the possibility that the involvement of parasitic plants

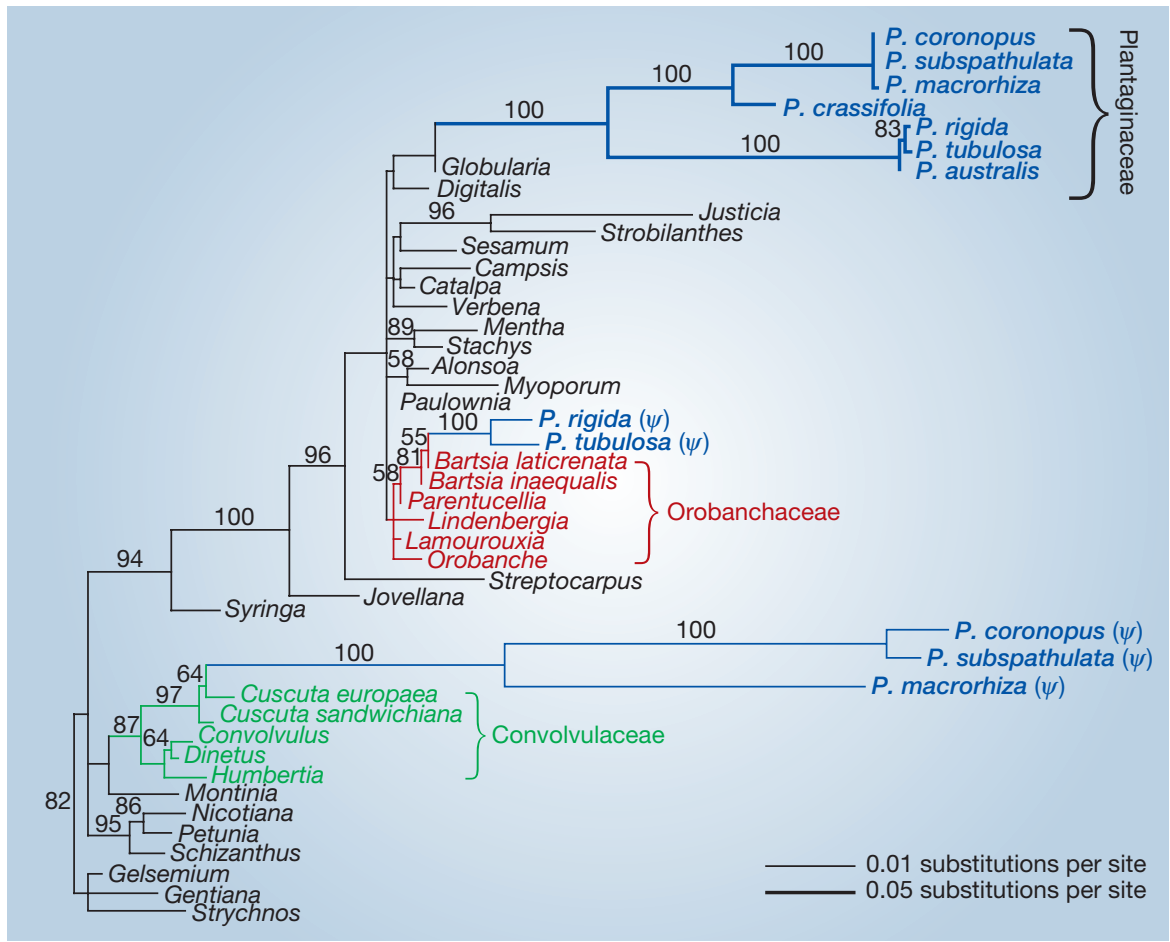

Figure 1 Phylogenetic evidence for two horizontal transfer events of the gene atp1 into Plantago (blue). Seven Plantago atp1 genes at the top of the maximum-likelihood tree are intact, vertically transmitted, and rapidly evolving (scale reduced by $80 \%$ ). The other two sets of Plantago atp1 genes are pseudogenes $(\psi)$ acquired from parasitic plants in the Orobanchaceae (red) and Convolvulaceae (green). Bootstrap values of over $50 \%$ are shown. For methods, see supplementary information.

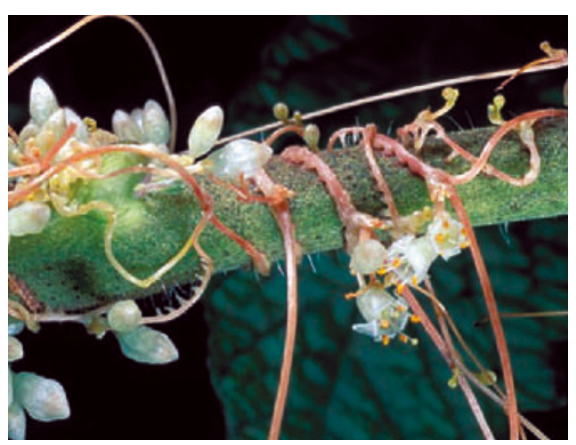

Figure $2 \mathrm{~A}$ parasitic dodder (Cuscuta californica) in flower, with its haustoria penetrating a host tomato plant.

as donors is fortuitous - for example, some biological vector (such as pollen, fungus, a bacterium or virus, or an insect) may have caused indirect transfer. But our directtransfer hypothesis is more parsimonious and is supported by the evidence available.

The parasitic lifestyle has evolved roughly a dozen times in flowering plants and there are more than 4,000 species of parasitic plants ${ }^{12}$, providing plenty of opportunity for horizontal gene transfer not only from parasite to host, but also from host to parasite ${ }^{3}$. Parasites with narrow host ranges may exchange genes frequently with their hosts. Those with broad host ranges (dodders, for example) may also act as vectors for gene transfer by directly bridging unrelated host species. In addition to parasitism, other vectors, and non-vectored mechanisms such as grafting, may promote horizontal gene transfer during plant evolution.
Jeffrey P. Mower, Saša Stefanović*, Gregory J. Young, Jeffrey D. Palmer Department of Biology, Indiana University, Bloomington, Indiana 47405, USA

e-mail:jpalmer@bio.indiana.edu

${ }^{\star}$ Present address: Department of Biology,

University of Toronto at Mississauga,

Mississauga, Ontario L5L 1C6, Canada

1. Bergthorsson, U., Adams, K. L., Thomason, B. \& Palmer, J. D. Nature 424, 197-201 (2003).

2. Won, H. \& Renner, S. S. Proc. Natl Acad. Sci. USA 100 10824-10829 (2003).

3. Davis, C. C. \& Wurdack, K. J. Science 305, 676-678 (2004)

4. Rønsted, N., Chase, M. W., Albach, D. C. \& Bello, M. A. Bot. J. Linn. Soc. 139, 323-338 (2002).

5. Yuncker, T. G. Mem. Torrey Bot. Club 18, 111-331 (1932).

6. Tessene, M. F. Mich. Bot. 8, 72-104 (1969).

7. Molau, U. Opera Bot. 102, 1-99 (1990).

8. Luteyn, J. Páramos: A Checklist of Plant Diversity, Geographical Distribution, and Botanical Literature (New York Botanical Garden Press, Bronx, New York, 1999).

9. Hosford, R. M. Bot. Rev. 33, 387-406 (1967).

10. Marcone, C., Hergenhahn, F., Ragozzino, A. \& Seemüller, E. J. Phytopathol. 147, 187-192 (1999).

11. Haupt, S., Oparka, K. J., Sauer, N. \& Neumann, S. J. Exp. Bot. 52, 173-177 (2001).

12. Nickrent, D. L. et al. in Molecular Systematics of Plants II (eds Soltis, D. E., Soltis, P. S. \& Doyle, J. J.) 211-241 (Kluwer, Boston, 1998).

Supplementary information accompanies this communication on Nature's website.

Competing financial interests: declared none.

\section{brief communications arising online}

\section{www.nature.com/bca}

Evolution: How do characters evolve?

A. Purvis (doi:10.1038/nature03092)

Reply: R. E. Ricklefs (doi:10.1038/nature03093) 